Bull. Mater. Sci., Vol. 38, No. 3, June 2015, pp. 645-651. (c) Indian Academy of Sciences.

\title{
Low power optical limiting studies on nanocrystalline benzimidazole thin films prepared by modified liquid phase growth technique
}

\author{
P A PRAVEEN ${ }^{1}$, S P PRABHAKARAN ${ }^{1}$, R RAMESH BABU ${ }^{1, *}$, K SETHURAMAN ${ }^{2}$ \\ and K RAMAMURTHI ${ }^{3}$ \\ ${ }^{1}$ Crystal Growth and Thin Film Laboratory, Department of Physics, Bharathidasan University, Tiruchirappalli 620024 , \\ Tamilnadu, India \\ ${ }^{2}$ School of Physics, Madurai Kamaraj University, Madurai 625 021, Tamilnadu, India \\ ${ }^{3}$ Crystal Growth and Thin Film Laboratory, Department of Physics and Nanotechnology, SRM University, \\ Kattankulathur 603 203, Kancheepuram, Tamilnadu, India
}

MS received 4 July 2014; revised 25 August 2014

\begin{abstract}
In the present work, benzimidazole (BMZ), a well known nonlinear optical material, thin films were deposited using the modified liquid phase growth technique at different solution temperatures. The prepared samples were subjected to spectral, structural and surface analyses. Linear optical properties and third-order optical nonlinearity of the deposited BMZ thin films were analysed using UV-visible-NIR spectrum and $Z$-scan technique, respectively. The experimental results show that the BMZ films exhibit reverse saturable absorption and positive nonlinearity at $650 \mathrm{~nm}$ CW laser of power $5 \mathrm{~mW}$. The measured third-order nonlinear susceptibility of the films is about $10^{-10} \mathrm{esu}$. Optical limiting studies were performed using the same laser source and the potentiality of the BMZ films is reported.
\end{abstract}

Keywords. Organic thin films; liquid phase growth; third-order nonlinear susceptibility; optical limiting.

\section{Introduction}

Organic molecules are of considerable interest owing to their extensive design possibilities and the better performance than that of their inorganic counterparts. Especially, organic materials with aromatic rings are considered as a potential class of nonlinear optical (NLO) materials due to their nonresonant high optical nonlinearity, fast response time and high optical damage threshold. ${ }^{1-3}$ They are the key materials for numerous photonic applications like signal processing, switching, frequency generation, optical data storage, optical communication and image processing. ${ }^{4,5}$ Also, organic materials with large third-order optical nonlinearities are widely considered for the third harmonic generation (THG) effects which is a promising mechanism for high bandwidth all-optical switching and processing devices. ${ }^{6}$

Benzimidazole (BMZ), a heterocyclic aromatic organic compound, well known for its antibacterial and antifungal activities. ${ }^{7}$ However, for the last two decades much attention have been paid towards material science-related research on BMZ because of its $\pi$-conjugated electronic system. In particular, several reports are available on second harmonic generation (SHG) effects of this material and its SHG efficiency is 4.5 times better than that of the standard KDP material. ${ }^{8,9}$ Although the title compound and its derivatives were investigated for a variety of applications, only a very few reports are

\footnotetext{
*Author for correspondence (rampap2k@yahoo.co.in)
}

available on the third-order NLO properties. Those reports mainly concern on structure-property relation and studied in solution form using high power pulsed lasers. ${ }^{10-13}$ However, for THG-related applications thin film structures are often considered as a suitable form since they provide accurate measurements of NLO effects and they are suitable for fabricating planar wave guides. ${ }^{14}$ In the past, $Z$-scan studies of the title compound with Mn metal ions are reported by our group. ${ }^{15}$ However it is not possible to obtain stable undoped BMZ crystalline films using the conventional deposition techniques. Hence, in the present study by using the modified liquid-phase growth technique, the undoped nanocrystalline BMZ thin films were deposited for the first time. In addition, the films were subjected to $Z$-scan to study the third-order nonlinearity and optical limiting studies to analyse the limiting ability of the prepared BMZ thin films.

\section{Experiments}

\subsection{Materials and methods}

Liquid phase growth (LPG) apparatus is a simple and an efficient technique to grow single crystalline organic semiconducting thin films. ${ }^{16}$ In the present work, we made some modifications in the LPG experimental setup in order to suit the method for BMZ thin film deposition and the schematic of the modified LPG is shown in figure 1. In the modified LPG technique, to obtain a temperature gradient for growth, 


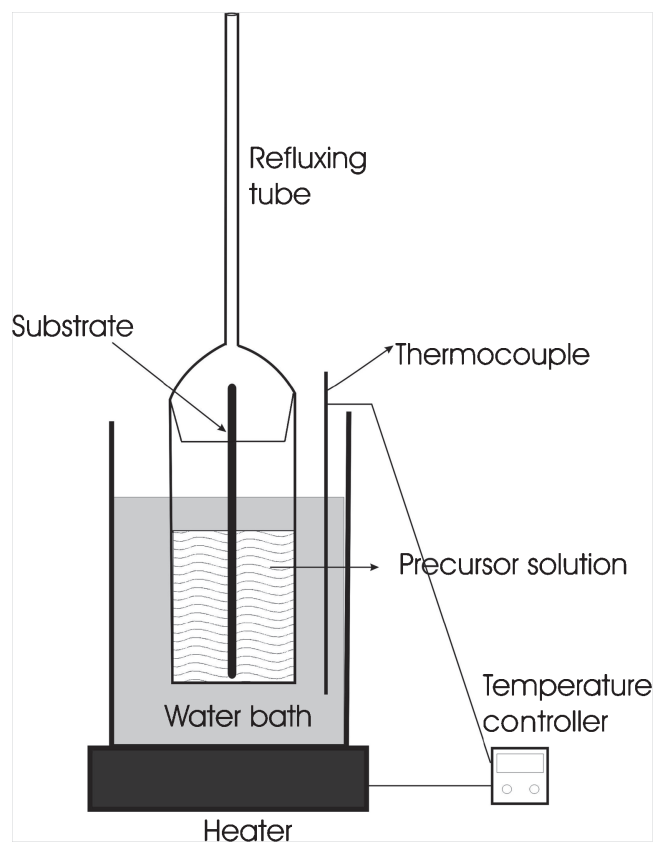

Figure 1. Modified LPG apparatus.

instead of fitting the substrate with metal plate here it was half-immersed into the solution and the temperature was applied only to the solution region. This offers more deposition area and reduces the effort of fitting glass substrates in metal plate. Likewise, thin aluminium sheet placed around the substrate, to control the number of molecules striking the substrate in LPG, was removed in the present case. Because the solute is added into the solution with low molar concentration, there is no need to control it specifically. In addition, our apparatus was equipped with a refluxing tube to control the rate of evaporation of volatile solvents like ethanol. Further, modified LPG offers various parameters such as temperature, rate of evaporation of solvent, $\mathrm{pH}$ of the solution, etc., to optimize the thin film deposition. For instance, thickness of the depositing thin film can be easily varied by adjusting the concentration of the solution and/or temperature of the water/oil bath. Analytical grade chemicals and reagents were used for the preparation of BMZ thin films. Microscopic glass plates were used as substrates and they were cleaned by the procedure as described elsewhere. ${ }^{17}$ Initially substrates were immersed into the $25 \%$ Aquaregia solution in water for $30 \mathrm{~min}$ to etch the surface slightly. Then they were washed with soap solution to remove the acidic- and grease-like residuals. Finally, the substrates were ultrasonically treated using acetone and water for $10 \mathrm{~min}$. The wellcleaned substrates were dried at $50^{\circ} \mathrm{C}$ in a hot air oven. The precursor BMZ solution was prepared using ethanol as solvent with $0.1 \mathrm{M}$ concentration. The solution temperature was varied from room temperature (RT, $29^{\circ} \mathrm{C}$ ) to $70^{\circ} \mathrm{C}$ and thin films of BMZ were deposited for three different temperatures $\left(50,60\right.$ and $\left.70^{\circ} \mathrm{C}\right)$. No films were formed at temperature less than $50^{\circ} \mathrm{C}$ and the solvent evaporation was very high for the temperatures more than $70^{\circ} \mathrm{C}$, hence the temperature range was fixed in between 50 and $70^{\circ} \mathrm{C}$. Thin films of $\mathrm{BMZ}$ were deposited by the immersion of two fused substrates into the corresponding solution for an hour. Then the substrates with films were removed from the solution, dried in a hot air oven for $2 \mathrm{~min}$ and cleaned with hot water.

\subsection{Characterization studies}

The optical transparency of deposited BMZ thin films was analysed by UV-Vis-NIR transmission spectrum in the region 300-1100 nm (Perkin Elmer Lambda 35). Surface topography of the deposited thin films was analysed using A.P.E. Research A 100 atomic force microscope (AFM) operated at non- contact mode. Thickness of the films was measured by the simple air wedge technique. In this method, BMZ film deposited on a glass substrate was placed on a microscopic glass plate in order to form a wedge. ${ }^{18}$ When this was illuminated with a $2 \mathrm{~mW} \mathrm{CW}$ laser, alternate dark and bright fringes were observed. By calculating the number of fringes per unit length one can calculate the thickness $(t)$ of the sample using the relation

$$
t=\frac{l \lambda}{2 \Delta X}
$$

where $l$ is the length of the sample illuminated, $\lambda$ the wavelength of source and $\Delta X$ the number of fringes per unit length. The IR absorption of BMZ powders scratched from the deposited thin films was characterized between 400 and $4000 \mathrm{~cm}^{-1}$ and is compared with the previous report on the title compound in powder form. ${ }^{19}$ Powder X-ray diffraction (PXRD) pattern of deposited films was recorded using XPERT-PRO X-ray diffractometer to analyse the crystalline nature.

Third-order NLO properties of deposited thin films were studied by the $Z$-scan technique using a $5 \mathrm{~mW} \mathrm{CW}$ diode laser of Gaussian profile at $650 \mathrm{~nm} .{ }^{20}$ A lens of focal length $15 \mathrm{~cm}$ was used to focus the laser beam on the sample. The spot size of the laser beam at the focus was calculated and it was found to be $42.62 \mu \mathrm{m}$. The corresponding Rayleigh length $\left(Z_{\mathrm{R}}\right)$ was calculated as $6.835 \mathrm{~mm}$. The thickness $(t)$ of the films are ranging from 108 to $162 \mathrm{~nm}$, which satisfies the critical condition $Z_{\mathrm{R}} \gg t$ and they could be treated as a thin medium. ${ }^{21}$ In $Z$-scan there are two possible configurations: closed aperture scan, where the laser beam transmitted through the sample was allowed to pass through an aperture placed in the far field and it was measured using a photodetector connected with digital power meter. In open aperture $Z$-scan, the aperture was replaced by a lens to collect the entire laser beam transmitted through the sample. From the open aperture $Z$-scan measurements, it is found that BMZ thin films exhibit reverse saturable absorption (RSA) behaviour. Hence, their ability towards optical limiting investigation is carried out using the same laser with slight modification, a polarizer was placed in front of the convex lens in order to vary the input intensity, in the $Z$-scan set-up. 


\section{Results and discussion}

The IR absorption of BMZ powders from BMZ films deposited at $60^{\circ} \mathrm{C}$ is shown in figure 2. Broad absorption appeared between 2300 and $3400 \mathrm{~cm}^{-1}$ is due to the presence of aromatic $\mathrm{C}-\mathrm{H}$ and $\mathrm{N}-\mathrm{H}$ bands. The sharp absorption peaks appeared at 1465 and $1409 \mathrm{~cm}^{-1}$ can be attributed to $\mathrm{C}=\mathrm{C}$ stretching vibrations and the peaks appeared between the region 1150 and $745 \mathrm{~cm}^{-1}$ are due to the inplane and out of plane vibrations of $\mathrm{C}-\mathrm{H}$ band. Peaks appeared at 1764 and $627 \mathrm{~cm}^{-1}$ are due to $\mathrm{N}-\mathrm{H}$ inplane and $\mathrm{N}-\mathrm{H}$ out of plane vibrations, respectively. The strong absorption at 1241 and $420 \mathrm{~cm}^{-1}$ is due to $\mathrm{C}-\mathrm{C}$ stretching and $\mathrm{C}-\mathrm{C}-\mathrm{C}$

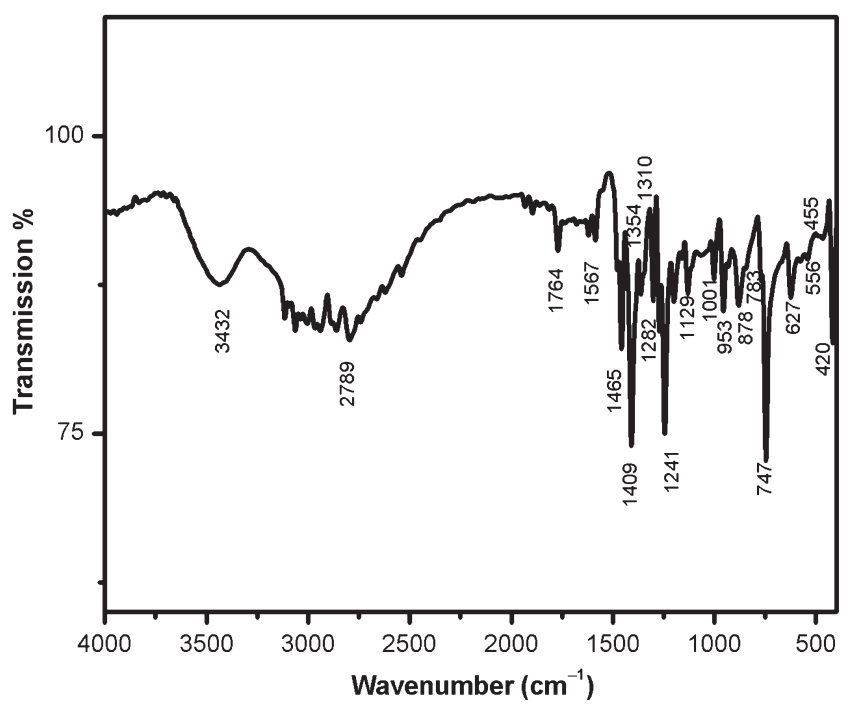

Figure 2. FTIR spectrum of BMZ film prepared at $60^{\circ} \mathrm{C}$. out of plane bending, respectively. Further, no peak shifts appeared or other organic impurity peaks related to ethanol or $\mathrm{OH}$ appeared. This ensures the purity of the deposited films. Also, there are no significant changes observed in the FTIR spectrum of the films deposited at different solution temperatures.

The surface topography of the films varies with different solution temperatures. The size of the grains (figure 3) decreases for the films deposited at $60^{\circ} \mathrm{C}$ and bulk adsorption of particles have been observed for the films deposited at $70^{\circ} \mathrm{C}$ compared to the films deposited at $50^{\circ} \mathrm{C}$. This in turn confirms the role of solution temperatures in film deposition. Calculated root mean square (RMS) roughness, skewness and kurtosis values of the BMZ films are given in table 1 . Hence the values of skewness is negative and kurtosis is less than 3 , it is concluded that all the films posses predominant valley and have a flat surface. ${ }^{22}$ From table 1 , it is evident that the flatness and skewness increase for the films deposited at $60^{\circ} \mathrm{C}$, means that, the films deposited at $60^{\circ} \mathrm{C}$ are more planar and homogenous than the films deposited at 50 and $70^{\circ} \mathrm{C}$.

The XRD patterns of BMZ films deposited at different solution temperatures are shown in figure 4. XRD patterns reveal that BMZ films prepared at different solution

Table 1. Surface topographical parameters of BMZ thin films.

\begin{tabular}{lccc}
\hline $\begin{array}{l}\text { Sample preparation } \\
\text { temperature }\left({ }^{\circ} \mathrm{C}\right)\end{array}$ & $\begin{array}{c}\text { RMS } \\
(\mathrm{nm})\end{array}$ & $\begin{array}{c}\text { Skewness } \\
(\text { arb. })\end{array}$ & $\begin{array}{c}\text { Kurtosis } \\
(\operatorname{arb} .)\end{array}$ \\
\hline 50 & 22.8 & -0.50 & 2.93 \\
60 & 20.9 & -0.26 & 2.17 \\
70 & 28.3 & -0.42 & 2.75 \\
\hline
\end{tabular}
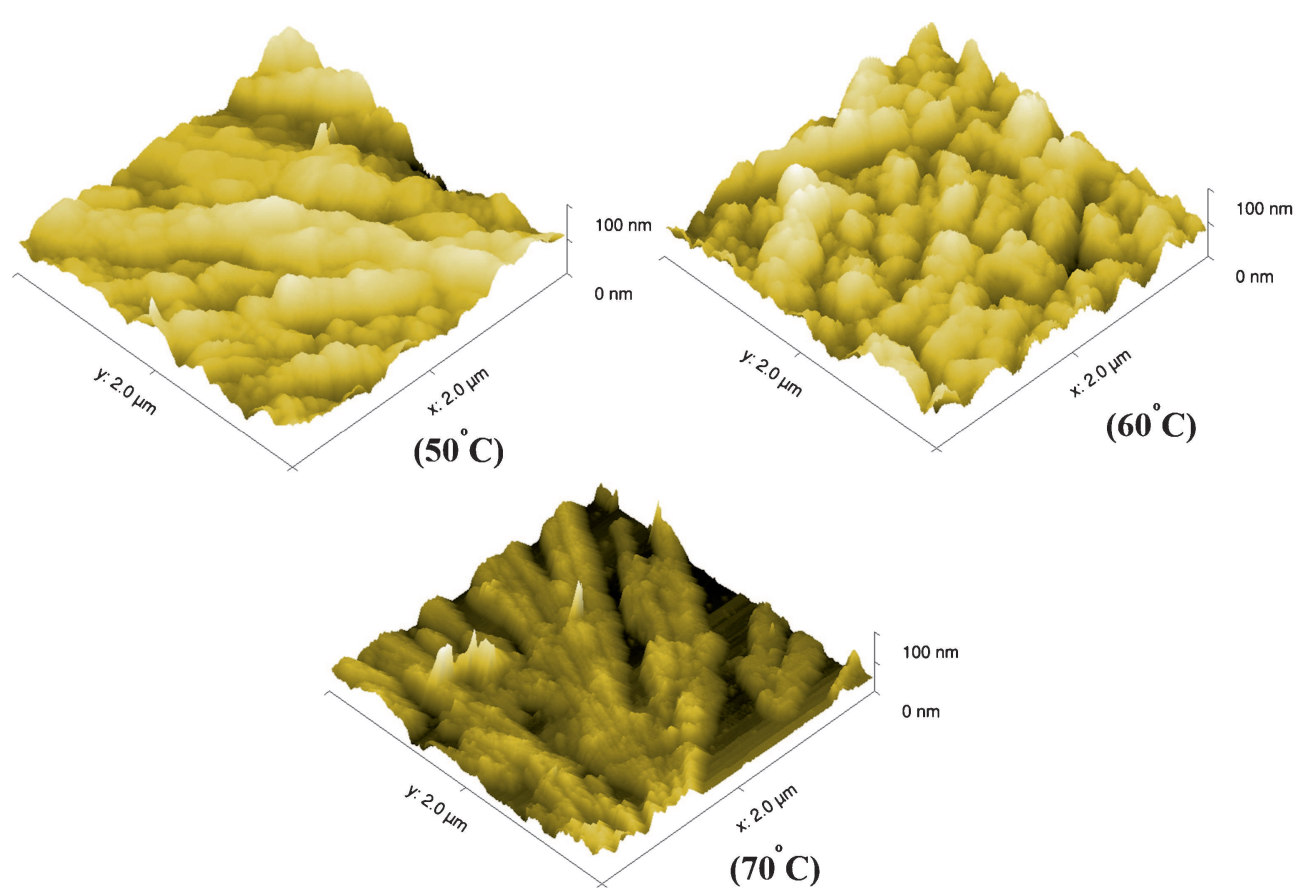

Figure 3. AFM images of BMZ films. 


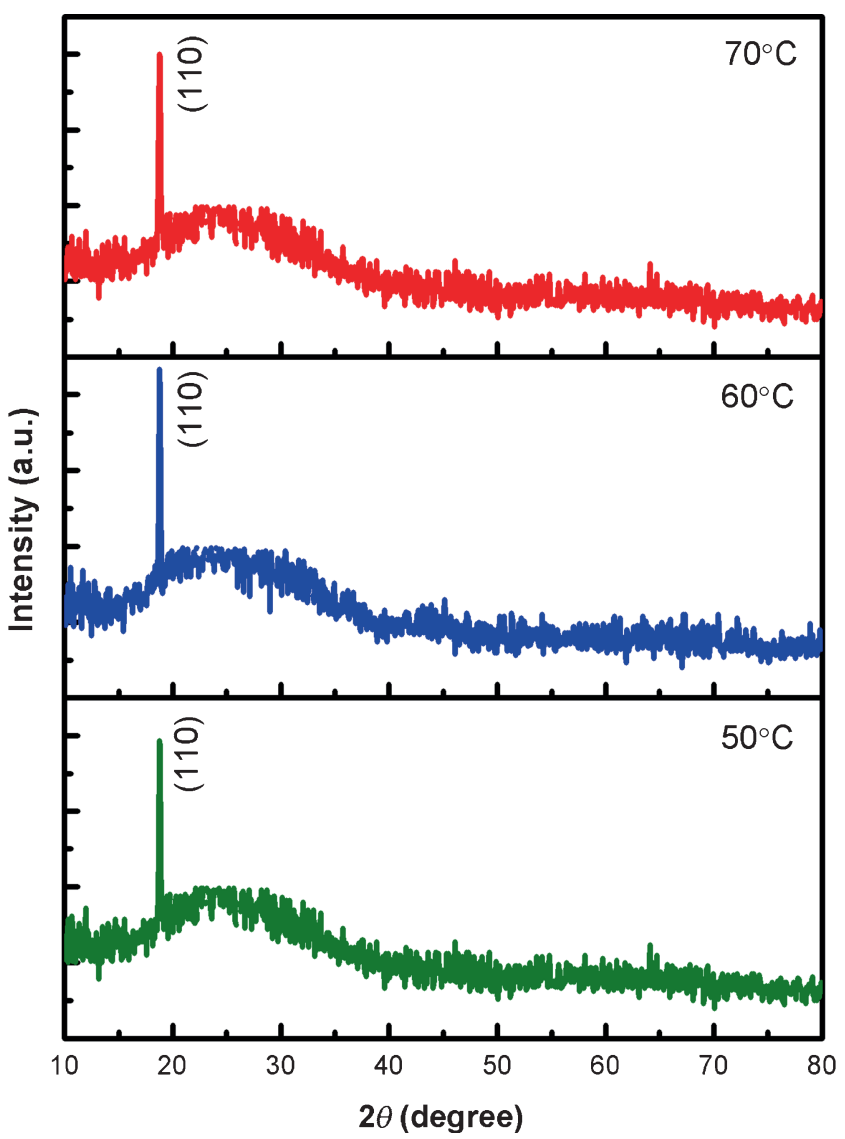

Figure 4. X-ray diffraction patterns of BMZ thin films.

temperatures contain only a single peak corresponding to (110) plane. ${ }^{8,9}$ This is because the molecules tend to arrange only in (110) plane and confirms the single crystalline nature of the films. The crystallite size of the BMZ films was calculated using the well known Debye-Scherrer's formula as

$$
D=\frac{0.9 \lambda}{\beta \cos \theta} .
$$

The calculated crystallite size with respect to solution temperatures is shown in figure 5. The results are similar to the patterns observed in AFM images. The variations in crystallite size confirm the contribution of solution temperature to the grain growth mechanism in thin films. ${ }^{23,24}$ With increasing temperature more number of nucleation sites can be possible and this in turn reduces the crystallite size. But at $70^{\circ} \mathrm{C}$ evaporation of ethanol leads to rapid adsorption results an increase in crystallite size.

Although no significant variations are observed in FTIR and XRD analyses of the deposited BMZ thin films, variation in thickness has been observed. This contributes to the linear and NLO properties of the films. The calculated thickness values of the films are listed in table 2 . The variation in thickness with respect to solution temperature is due to the deposition process. During the deposition of BMZ thin films, there is no possibility of chemical reaction and physisorption is the reason for the deposition of material as thin films. ${ }^{25}$

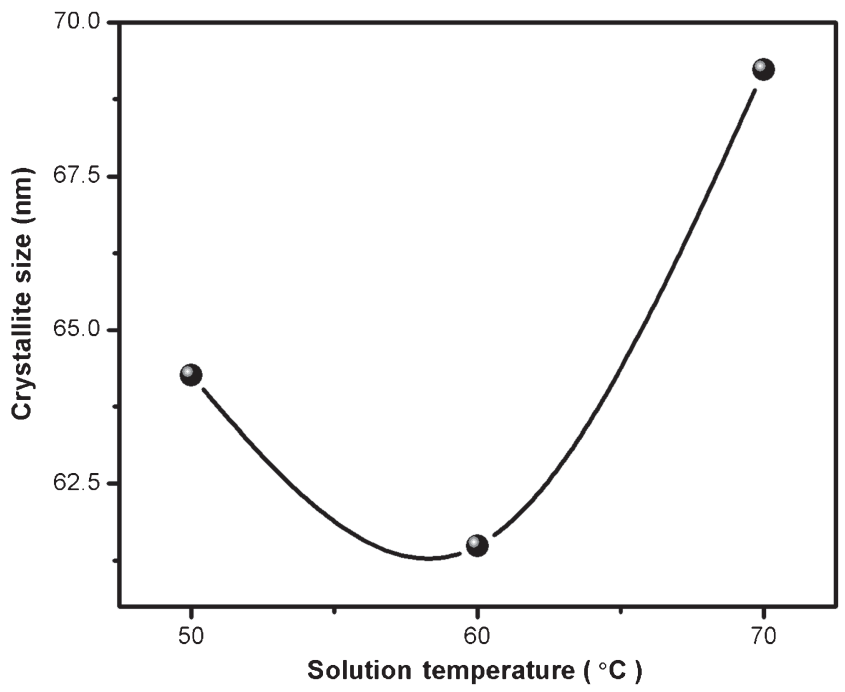

Figure 5. Crystallite size of BMZ thin films.

Table 2. Thickness of BMZ thin films.

\begin{tabular}{lc}
\hline $\begin{array}{l}\text { Sample preparation } \\
\text { temperature }\left({ }^{\circ} \mathrm{C}\right)\end{array}$ & $\begin{array}{c}\text { Thickness } \\
(\mathrm{nm})\end{array}$ \\
\hline 50 & 108.3 \\
60 & 144.4 \\
70 & 162.5
\end{tabular}

Physisorption of molecules are due to weak van der Waals forces and often these forces are not enough to induce a nucleation. Therefore, supply of thermal energy is necessary to induce interactions between the molecules and substrate. ${ }^{26}$ In the present work, at temperatures lower than $50^{\circ} \mathrm{C}$ no films were obtained due to the insufficient energy supplied to the molecules to nucleate on the substrate. When the solution temperature is about $50^{\circ} \mathrm{C}$ the molecules gain sufficient energy to nucleate and can form grains on the substrate. In principle, energy gained by the molecules are directly proportional to the deposition rate and thickness of the films, i.e., with increasing temperature more number of molecules tend to adsorb on the surface of the substrate and this can be evident from the thickness of the films deposited at 60 and $70^{\circ} \mathrm{C}$.

The transmittance spectra of BMZ thin films were recorded in the wavelength range from 300 to $1100 \mathrm{~nm}$ and are shown in figure 6. From the figure, it can be clearly seen that the films deposited at $60^{\circ} \mathrm{C}$ show higher transmittance percentage than that of the films deposited at 50 and $70^{\circ} \mathrm{C}$. Variation in transmittance might be due to the variation in grain size of the films and the films with lower grain size show higher transmission percentage. ${ }^{27}$ Further, with increasing optical path length, i.e., with increasing thickness, the optical scattering losses may also increase. That might also contribute to the decrement of transmittance in the case of films deposited at $70^{\circ} \mathrm{C}$ compared to the films deposited at 


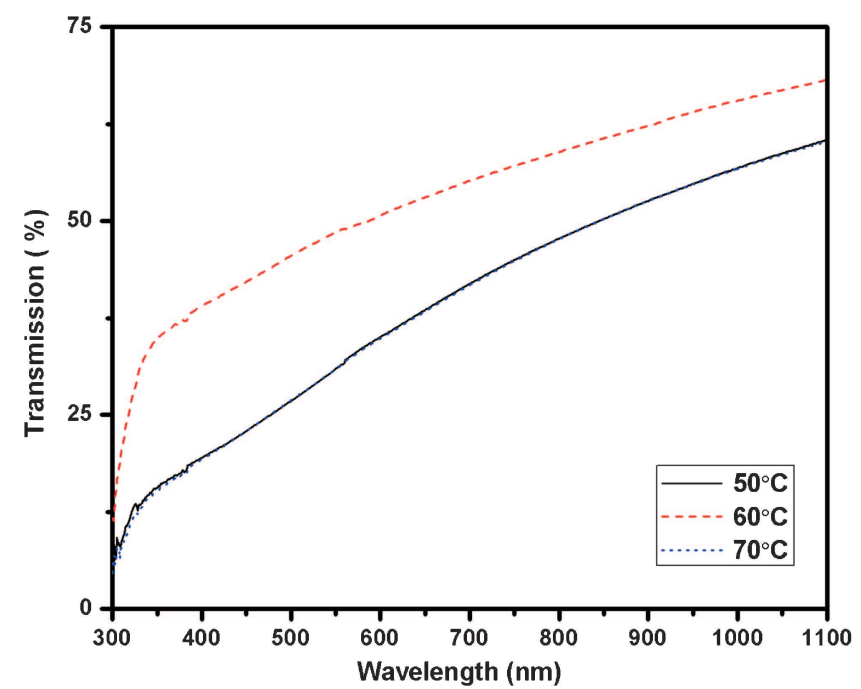

Figure 6. Transmittance spectrum of BMZ thin films.

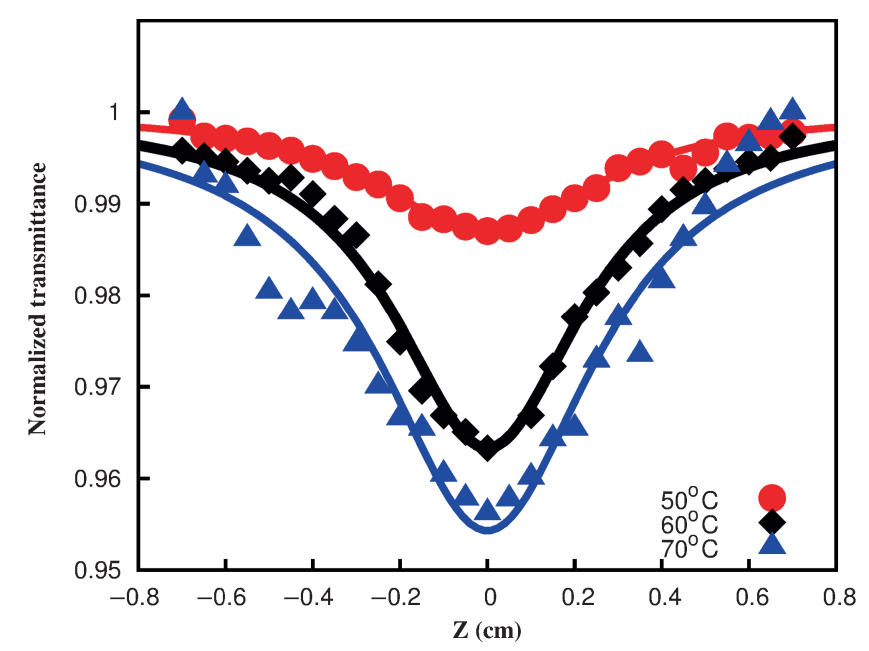

Figure 7. Open aperture $Z$-scan curve for BMZ thin films.

$60^{\circ} \mathrm{C}$. For BMZ thin films with decreasing grain size the fundamental absorption edge has been red shifted, ${ }^{28}$ indicating the possibility of electronic transition at lower energy region.

Figures 7 and 8 show the open and closed aperture $Z$-scan curves of the deposited BMZ thin films, respectively. The obtained open aperture curves are symmetric with respect to focus $(Z=0)$ where it has minimum transmittance which confirms the nonlinear absorption like RSA. ${ }^{29}$ When the transmittance was measured for closed aperture $Z$-scan, the detector is sensitive to both nonlinear refraction and nonlinear absorption. Therefore, the closed aperture transmittance was divided by corresponding open aperture data to obtain the pure nonlinear refraction. ${ }^{21}$ In figure 8 , the prefocal transmittance minimum (valley) followed by a postfocal transmittance maximum (peak) indicates that the refractive nonlinearity is positive, i.e., self-focusing. This is due to local variation of refractive index with temperature. ${ }^{30}$

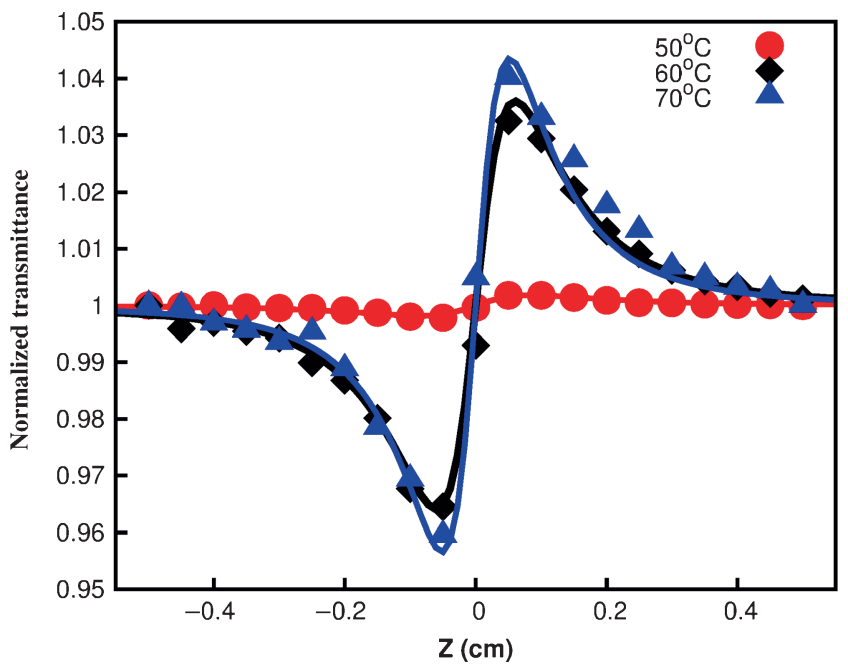

Figure 8. Closed aperture $Z$-scan curve for BMZ thin films.

The nonlinear absorption coefficient $(\beta)$ is calculated by fitting the relation ${ }^{21}$

$$
T(z)=1-\frac{q_{0}}{2 \sqrt{2}\left(1+z^{2} / z_{0}^{2}\right)}
$$

to the experimental data. Here, $z$ is the position of the sample with respect to focal position, $q_{0}=\beta I_{0} L_{\text {eff }}, I_{0}$ is the laser intensity at focus, $L_{\text {eff }}$ the effective thickness of the sample and can be calculated from the equation $L_{\text {eff }}=\left(1-\mathrm{e}^{-\alpha \mathrm{L}}\right) / \alpha$ and $\alpha$ is the absorption coefficient of the film. Similarly, the nonlinear refractive index is calculated by fitting the relation

$$
T(z)=1+\frac{4 \Delta \phi_{0}\left(z / z_{0}\right)}{\left[\left(z / z_{0}^{2}\right)+9\right]\left[\left(z / z_{0}^{2}\right)+1\right]},
$$

where $\Delta \phi_{0}=n_{2} K I_{0} L_{\text {eff }}$ and $n_{2}$ is the nonlinear refractive index. The nonlinear absorption and refractive index were used to calculate the real $\operatorname{Re} \chi^{(3)}$ and imaginary $\operatorname{Im} \chi^{(3)}$ parts of the third-order nonlinear susceptibility using the relation ${ }^{21}$

$$
\operatorname{Im} \chi^{(3)}=\frac{n^{2} \varepsilon_{0} c \beta}{K}
$$

and

$$
\operatorname{Re} \chi^{(3)}=4 / 3\left(n^{2} \varepsilon_{0} c n_{2}\right)
$$

where $c$ is the speed of light in vacuum, $\varepsilon_{0}$ the free space permittivity and $n$ the linear refractive index of the material and $K=(2 \pi / \lambda)$. The absolute values of third-order nonlinear susceptibilities of BMZ films were calculated using

$$
\left.\left|\chi^{(3)}\right|=\left[\left(\operatorname{Re} \chi^{(3)}\right)^{2}+\operatorname{Im} \chi^{(3)}\right)^{2}\right]^{1 / 2} .
$$

Since the films have different thicknesses, their potentiality was estimated by calculating the figure of merit for each sample with respect to their linear absorption coefficient. ${ }^{31}$ Table 3 provides third-order NLO data of BMZ thin films. From the table, it is observed that the major contribution 
Table 3. Third-order nonlinear optical data of BMZ thin films.

\begin{tabular}{lrrrrrr}
\hline $\begin{array}{l}\text { Sample preparation } \\
\text { temperature } \\
\left({ }^{\circ} \mathrm{C}\right)\end{array}$ & $\begin{array}{r}\operatorname{Im} \chi^{(3)} \\
10^{-10} \\
(\mathrm{esu})\end{array}$ & $\begin{array}{r}\operatorname{Re} \chi^{(3)} \\
10^{-10} \\
(\mathrm{esu})\end{array}$ & $\begin{array}{c}\chi^{(3)} \\
10^{-10} \\
(\mathrm{esu})\end{array}$ & $\begin{array}{c}\chi^{(3) / \alpha} \\
10^{-17} \\
(\mathrm{esu})\end{array}$ & $\begin{array}{c}n_{2} \\
10^{-7} \\
\left(\mathrm{~cm}^{2} \mathrm{~W}^{-1}\right)\end{array}$ & $\begin{array}{c}\beta \\
\left(\mathrm{cm} \mathrm{W}^{-1}\right)\end{array}$ \\
\hline 50 & 5.71 & 2.16 & 7.87 & 8.95 & 0.23 & 0.80 \\
60 & 9.65 & 23.18 & 32.83 & 74.84 & 2.53 & 1.36 \\
70 & 11.68 & 23.27 & 34.96 & 59.32 & 2.54 & 1.64 \\
\hline
\end{tabular}

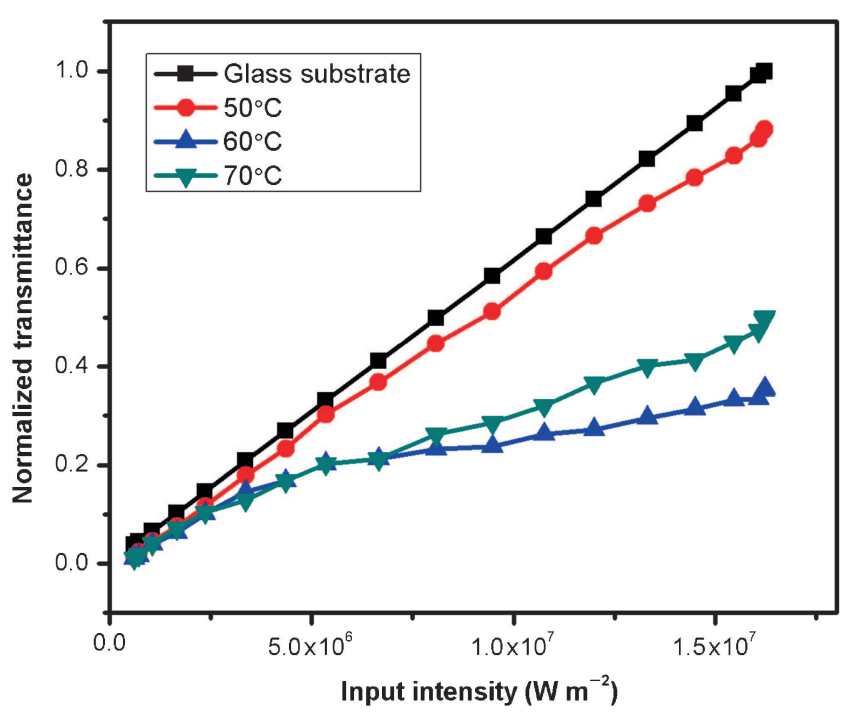

Figure 9. Optical limiting properties of BMZ thin films.

of nonlinear susceptibility comes from nonlinear refraction of the material because the real part the of third-order nonlinear susceptibility is greater than that of imaginary part. The large nonlinear refractive values could be attributed to the thermal effects which is a common phenomenon in $\mathrm{CW}$ regime. Further, the RSA behaviour observed in OA scans can be explained using a five level energy model. ${ }^{32}$ In which, the absorption of photons between energy levels is either due to two photon absorption (TPA) or excited state absorption (ESA). In the case BMZ thin films, RSA can be caused by ESA which is a common behaviour in $\mathrm{CW}$ regime. The thermal effects due to $\mathrm{CW}$ illumination increases the population of excited state there by the molecule gets excited to the next energy level. Also, the organic molecules have quite long triplet state, favouring the intersystem crossing (ISC) in the molecule which leads to observation of RSA.

From the open aperture $Z$-scan curves, it is clear that the films exhibit RSA behaviour at $650 \mathrm{~nm}$. These types of films could be effectively employed for optical limiting applications. ${ }^{31}$ Optical limiting with low power lasers is also very important in the sense that even a diode laser of power $2 \mathrm{~mW}$ can damage the human eyes. ${ }^{33}$ The experimental setup for analysing optical limiting behaviour is similar to that of closed aperture $Z$-scan setup except a polarizer is placed in front of the focal lens to vary the input power on the sample. Variation of output intensity is recorded with and without the samples. The obtained values were normalized and it is plotted $v s$. input intensity which is shown in figure 9. Initially, the output intensity increases with the increase in input intensity and then the nonlinear medium starts to limit the output. Films prepared at $60^{\circ} \mathrm{C}$ show a better limiting behaviour than that of the films prepared at 50 and $70^{\circ} \mathrm{C}$. The variation in limiting effect is due to nonlinear scattering which varies with respect to the thickness of the thin films. ${ }^{34}$

\section{Conclusion}

Nanocrystalline BMZ thin films were deposited by a modified LPG technique at different solution temperatures. Optical and structural studies reveal good transmittance and nanocrystalline nature of the samples. FTIR spectrum analysis confirms the presence of BMZ functional groups. The nonlinear absorption can be attributed to reverse saturable absorption whereas nonlinear refraction leads to selffocusing in BMZ films. The nonlinear absorption coefficient $(\beta)$ and nonlinear refractive index $\left(n_{2}\right)$ of the films were calculated and the third-order nonlinear susceptibility is in the order of $10^{-10}$ esu. Further the optical limiting behaviour of the films was studied at $650 \mathrm{~nm}$ and it is found that the films show limiting behaviour in that region. In conclusion, the optical properties discussed in this work may be useful to develop a device for low power optical limiting and other third-order NLO-related applications.

\section{Acknowledgements}

We acknowledge the DST-PURSE program of School of Physics, Madurai Kamaraj University, for providing AFM facility. PA Praveen thanks the UGC-BSR Government of India, for financial assistance in the form of Research Fellowship in Science for Meritorious Students (F.4-1/2006/7197/2007(BSR)). He also thanks Mr M Sambath, Research Scholar, School of Physics, for his kind help in recording AFM images.

\section{References}

1. Sharafudeen K, Adithya A, Vijayakumar S, Sudheesh P, Kalluraya B and Chandrasekharan K 2011 Curr. Appl. Phys. 111089

2. Kiran A J, Lee H, Ravindra H, Dharmaprakash S, Kim K, Lim H and Rotermund F 2010 Curr. Appl. Phys. 101290 
3. Prasad P N and Williams D J (eds) 1991 Introduction to nonlinear optical effects in molecules and polymers (New York: John Wiley \& Sons Inc.) 1st ed

4. Costa $\mathrm{N}$ and Cartaxo A (eds) 2010 Advances in lasers and electrooptics (Croatia: INTECH) 1st ed

5. Sun W, Wang Z, Chen A, Kosilkin I, Bale D and Dalton L R 2011 Opt. Express 1911189

6. D'Amorea F, Zappettinia A, Facchinia G, Pietralungaa S, Martinellia M, Dell'Erbac C, Cunibertic C, Comorettoc D and Dellepiane G 2002 Synth. Met. 127143

7. Maxwell W and Brody G 1971 Appl. Microbiol. 21944

8. Vijayan N, Bhagavannarayana G, Budakoti G, Kumar B, Upadhyaya V and Das S 2008 Mater. Lett. 621252

9. Vijayan N, Ramesh Babu R, Gopalakrishnan R, Ramasamy P and Harrison W 2004 J. Cryst. Growth 262490

10. Rabbani M G and El-Kaderi H M 2011 Chem. Mater. 23 1650

11. Rabbani M G and El-Kaderi H M 2012 Chem. Mater. 24 1511

12. Lee D Y, Singh N, Kim M J and Jang D O 2011 Org. Lett. 13 3024

13. Zhao M, Samoc M and Prasad P N 1990 Chem. Mater. 2670

14. Thakur M and Meyler S 1985 Macromolecules 182341

15. Praveen P A, Ramesh Babu R, Prabhakaran S P and Ramamurthi K 2014 AIP Conf. Proc. 1591991

16. Yamao T, Miki T, Akagami H, Nishimoto Y, Ota S and Hotta S 2007 Chem. Mater. 193748

17. Shugar G and Ballinger J 1996 Chemical technicians ready reference handbook (New York: McGraw Hill)

18. Tyler F 1974 A laboratory manual of physics (London: Edward Arnold Limited) 4th ed
19. Mohan S, Sundaraganesan N and Mink J 1991 Spectrochim. Acta 47A 1111

20. Fei H, Wei Z, Yang Q, Che Y, Shen Y, Fu X and Qui L 1995 Opt. Lett. 201518

21. Kuzyk M G and Dirk C W (eds) 1998 Characterization techniques and tabulations for organic nonlinear materials (New York: Marcel Dekker, Inc.) 1st ed

22. Mendez-Vilas A and Diaz J (eds) 2007 Modern research and educational topics in microscopy (Badajoz: FORMATEX) vol. 2

23. Lokovic K S, Bergmann R B and Bill A 2010 Mater. Res. Soc. Symp. Proc. 1245 1245-A16-07

24. Fewster P F 1996 Rep. Prog. Phys. 591339

25. Ohring M 2001 Materials science of thin films (London: Academic Press)

26. Chopra K L 1969 Thin film phenomena (New York: McGrawHill) vol. 137

27. Ramana C, Smith R and Hussain O 2003 Phys. Status Solidi (A) $199 \mathrm{R} 4$

28. Prabhakaran S, Ramesh Babu R and Ramamurthi K 2013 Rev. Sci. Instrum. 84083907

29. Manaa H, Tuhl A, Samuel J, Al-Mulla A, Al-Awadi N A and Makhseed S 2011 Opt. Commun. 284450

30. Bahae M S, Said A A, Wei T H, Hagan D J and Stryland E W V 1990 IEEE J. Quant. Electron. 26760

31. Krishnakumar V, Shanmugam G and Nagalakshmi R $2012 \mathrm{~J}$. Phys. D: Appl. Phys. 45165102

32. Zafar S, Khan Z H and Khan M S 2014 Spectrochim. Acta Part A: Mol. Biomol. Spectrosc. 118852

33. Innocenzi P and Lebeau B 2005 J. Mater. Chem. 153821

34. Kovsh D I, Yang S, Hagan D J and Stryland E W V 1999 Appl. Opt. 385168 